\title{
Theoretical models for the quantification of lung injury using ventilation and perfusion distributions
}

\author{
B.S. Brook ${ }^{\mathrm{a}}$, C.M. Murphy ${ }^{\mathrm{b}}$, D. Breen ${ }^{\mathrm{c}}$, A.W. Miles ${ }^{\mathrm{b}}$, D.G. Tilley ${ }^{\mathrm{b}}$ and A.J. Wilson ${ }^{\mathrm{d} *}$ \\ ${ }^{a}$ School of Mathematical Sciences, University of Nottingham, Nottingham, UK; ${ }^{b}$ Department of \\ Mechanical Engineering, University of Bath, Bath, UK; ${ }^{c}$ Department of Anaesthesthetics, Royal \\ Hallamshire Hospital, Sheffield, UK; ${ }^{d}$ Department of Physics, University of Warwick, Coventry, UK
}

(Received 10 March 2007; final version received 21 March 2008)

\begin{abstract}
This paper describes two approaches to modelling lung disease: one based on a multicompartment statistical model with a log normal distribution of ventilation perfusion ratio $(\dot{V} / \dot{Q})$ values; and the other on a bifurcating tree which emulates the anatomical structure of the lung. In the statistical model, the distribution becomes bimodal, when the $\dot{V} / \dot{Q}$ values of a randomly selected number of compartments are reduced by $85 \%$ to simulate lung disease. For the bifurcating tree model a difference in flow to the left and right branches coupled with a small random variation in flow ratio between generations results in a log normal distribution of flows in the terminal branches. Restricting flow through branches within the tree to simulate lung disease transforms this log normal distribution to a bi-modal one. These results are compatible with those obtained from experiments using the multiple inert gas elimination technique, where log normal distributions of $\dot{V} / \dot{Q}$ ratio become bimodal in the presence of lung disease.
\end{abstract}

Keywords: modelling; lung disease; $\dot{V} / \dot{Q}$; log normal; tree

2000 Mathematics Subject Classification: 92C30; 46F10; $05 \mathrm{C} 05$

\section{Introduction}

Models of the respiratory system which include lung disease ideally require mathematical descriptions of both the anatomical and physiological impacts of the disease, and in particular, the impact the disease has on gas exchange within the lung. The primary determinant of gas exchange efficiency in the lung is the matching of alveolar ventilation with blood flow through the alveolar capillaries and the heterogenous distribution of ventilation $(\dot{V})$ and perfusion $(\dot{Q})$ across the lung. This has been studied by a variety of experimental techniques including: multiple inert gas elimination technique (MIGET) [26], isotope imaging [4], positron emission tomography (PET; [16,21,22]) and fluorescent microspheres [1-3,9,20]. In order to achieve a better understanding of how the anatomical structure of the lung relates to gas exchange, a number of mathematical models of the lung have been proposed including 3D finite element models $[13,15]$, flow bifurcation models [28] and fractal geometries [5,9,17].

The majority of these models have been developed for normal lungs and to date little attention has been paid to the problem of modelling diseased lungs, in particular lungs with acute disease. For subjects with very severe acute lung disease who are admitted to

* Corresponding author. Email: adrian.wilson@warwick.ac.uk 
intensive care, it has been shown that the disease is spatially localized within the lung $[14,23]$. This suggests that acute lung disease, sometimes referred to as acute lung injury, can potentially be modelled as a spatially localized change in the $\dot{V} / \dot{Q}$ values found in the normal lung. Ventilation and perfusion distributions are commonly described in terms of a log normal function, since this allows a wide dynamic range in the value of the parameters without the presence of negative values, which are physiologically impossible. The parameter recovery algorithms used in the MIGET aim to provide ventilation and perfusion distributions that are log normally distributed for normal subjects [11,24]. When these parameter recovery algorithms are applied to the data obtained from MIGET on patients with chronic adult respiratory distress syndrome (ARDS) [7] or other pathologies [26] bimodal distributions result. A unimodal distribution in normal lungs which becomes bimodal in the presence of lung disease is compatible with the experimental finding that lung disease is spatially localized [14,23], but not uniquely so.

In this paper, we explore the use of log normal data series as a basis for modelling the distribution of $\dot{V} / \dot{Q}$ ratios in a multi-compartment lung. In addition, we propose a theoretical model of the lung based on a bifurcating tree structure that represents the conducting airways of the lung extending from the trachea to the terminal bronchioles as the basis for modelling lung disease. Irrespective of the aetiology of the lung disease, it ultimately affects gas exchange across the alveolar membrane, altering the $\dot{V} / \dot{Q}$ ratio which in turn can be regarded as effectively reducing the volume of gas and/or blood available to participate in gas exchange. Therefore, modifications to the values in the log normal data series or to the structure of the bifurcating tree potentially allow the effects of regional lung disease to be modelled.

\section{Theoretical models}

\subsection{Statistical models}

The log normal probability density function is characterized by two parameters $\mu$ and $\sigma$ and is given by:

$$
F(x \mid \mu, \sigma)=\frac{1}{x \sigma \sqrt{2 \pi}} \mathrm{e} \frac{-(\ln (x)-\mu)^{2}}{2 \sigma^{2}},
$$

and where the distribution is characterized by:

$$
\begin{gathered}
\text { Mean }=\mathrm{e}^{\mu+\frac{\sigma^{2}}{2}}, \\
\text { Median }=\mathrm{e}^{\mu}, \\
\text { Variance }=\mathrm{e}^{\left(2 \mu+2 \sigma^{2}\right)}-\mathrm{e}^{\left(2 \mu+\sigma^{2}\right)} .
\end{gathered}
$$

It can be seen that the mean of the distribution depends on both $\mu$ and $\sigma$. In order to construct a log normal distribution for ventilation, we can use the fact that the mean of the ventilation distribution is given by $E(x)=\dot{V}_{\text {tot }} / N \mathrm{ml} \mathrm{min}^{-1}$, where $\dot{V}_{\text {tot }}$ is the total ventilation and $N$ is the number of compartments used to model the lung. Using this and rearranging Equation (2) gives the parameter $\mu_{\dot{V}}$ :

$$
\mu_{\dot{V}}=\ln \left(\frac{\dot{V}_{\text {tot }}}{N}\right)-\frac{1}{2} \sigma_{\dot{V}}^{2}
$$


where $\mu_{\dot{V}}$ and $\sigma_{\dot{V}}$ are the parameters associated with the ventilation distribution. Replacing $\dot{V}_{\text {tot }}$ and $\sigma_{\mathrm{v}}$ in the above expression with $\dot{Q}_{\text {tot }}$ and $\sigma_{\dot{Q}}$ gives an equivalent expression for $\mu_{\dot{Q}}$, where the subscript $\dot{Q}$ indicates the perfusion distribution.

It is not clear at this stage how numerical values for the parameters $\sigma_{\dot{V}}$ and $\sigma_{\dot{Q}}$ for a normal lung can be assigned as they cannot, by definition, be measured or inferred directly. Wagner et al. [24] quote values of $\sigma_{\dot{V}}$ and $\sigma_{\dot{Q}}$ derived from distributions, where $\dot{V}$ and $\dot{Q}$ were expressed as a function of $\dot{V} / \dot{Q}$ (which we have denoted $\sigma_{\dot{V}} \mid \dot{V} / \dot{Q}$ and $\sigma_{\dot{Q}} \mid \dot{V} / \dot{Q}$, respectively) for a young normal male of $\sigma_{\dot{V}} \mid \dot{V} / \dot{Q}=0.35$ and $\sigma_{\dot{Q}} \mid \dot{V} / \dot{Q}=0.43$. However, in order to examine the effects of lung disease on log normal distributions, we need to know the values of $\sigma_{\dot{V}}$ and $\sigma_{\dot{Q}}$ for their native distributions (distributions of number of compartments as a function of ventilation or perfusion), rather than those obtained as a function of $\dot{V} / \dot{Q}$. There is clearly a relationship between the values of $\sigma_{\dot{V}}$ and $\sigma_{\dot{V}} \mid \dot{V} / \dot{Q}$ and between $\sigma_{\dot{Q}}$ and $\sigma_{\dot{Q}} \mid \dot{V} / \dot{Q}$. Establishing this relationship gives a potential way of determining numerical values for $\sigma_{\dot{V}}$ and $\sigma_{\dot{Q}}$.

In order to explore this relationship, Equation (1) was used to generate a series of distributions for $\dot{V}$ and $\dot{Q}$, using typical values of alveolar ventilation $\dot{V}_{T}$ and total perfusion $\dot{Q}_{T}$ of 5250 and $5000 \mathrm{ml} \mathrm{min}^{-1}$, respectively, whilst the values of $\sigma_{\dot{V}}$ and $\sigma_{\dot{Q}}$ were varied between 0.1 and 1 in steps of 0.1 . These distributions were combined using the method of West and Wagner [26] to give distributions of $\dot{V}$ and $\dot{Q}$ as a function of $\dot{V} / \dot{Q}$. For each combination of values of $\sigma_{\dot{V}}$ and $\sigma_{\dot{Q}}$, the values of $\sigma_{\dot{V}} \mid \dot{V} / \dot{Q}$ and $\sigma_{\dot{Q}} \mid \dot{V} / \dot{Q}$ were calculated. From the plots of these data (Figure 1) it can be seen that $\sigma_{\dot{Q}} \mid \dot{V} / Q$ varies approximately linearly with $\sigma_{\dot{V}}$ and $\sigma_{\dot{Q}}$ and that $\sigma_{\dot{V}} \mid \dot{V} / \dot{Q}$ varies linearly with $\sigma_{\dot{V}}$ and $\sigma_{\dot{Q}}$ for values of $\sigma_{\dot{V}}$ less than about 0.5 . It therefore follows that $\sigma_{\dot{V}} \mid \dot{V} / \dot{Q}$ and $\sigma_{\dot{Q}} \mid \dot{V} / \dot{Q}$ will vary linearly with $\left|\sigma_{\dot{V}}-\sigma_{\dot{Q}}\right|\left(=\Delta \sigma\right.$, Figure 2) for values of $\sigma_{\dot{V}}$ less than about 0.5 . Further processing of the data given in Figures 1 and 2 showed that the gradient of the relationship between $\sigma_{\dot{V}} \mid \dot{V} / \dot{Q}$ and $\left|\sigma_{\dot{V}}-\sigma_{\dot{Q}}\right|$ and $\sigma_{\dot{Q}} \mid \dot{V} / \dot{Q}$ and $\left|\sigma_{\dot{V}}-\sigma_{\dot{Q}}\right|$ varied linearly with the ratio $\dot{V}_{T} / \dot{Q}_{T}$ (Figure 3) and therefore, $\left|\sigma_{\dot{V}}-\sigma_{\dot{Q}}\right|$ can only be determined for a specific value of $\dot{V}_{T} / \dot{Q}_{T}$. The data in Figure 2 is for typical values of total ventilation $\left(\dot{V}_{T}\right)$ and total perfusion $\left(\dot{Q}_{T}\right)$ of 5250 and $5000 \mathrm{ml} \mathrm{min}^{-1}$, respectively, $\left(\dot{V}_{T} / \dot{Q}_{T}=1.05\right)$. Using these data with $\sigma_{\dot{V}} \mid \dot{V} / \dot{Q}=0.35$ and $\sigma_{\dot{Q}} \mid \dot{V} / \dot{Q}=0.43$ yielded an average value for $\left|\sigma_{\dot{V}}-\sigma_{\dot{Q}}\right|$ of 0.35 . Having obtained a value for $\left|\sigma_{\dot{V}}-\sigma_{\dot{Q}}\right|$, it was now necessary to determine a mean value of $\sigma\left(\left(\sigma_{\dot{V}}+\sigma_{\dot{Q}}\right) / 2\right)$, so that explicit values of $\sigma_{\dot{V}}$ and $\sigma_{\dot{Q}}$ could be determined. In order to determine the sensitivity of the
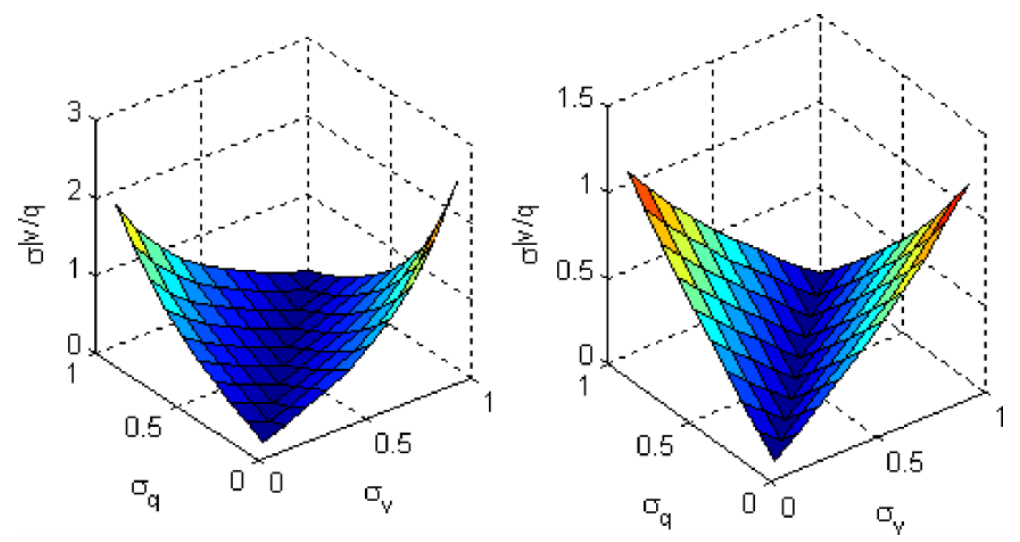

Figure 1. Plots showing the relationship between $\sigma_{\dot{V}}$ and $\sigma_{\dot{Q}}\left(\sigma_{\mathrm{v}}\right.$ and $\sigma_{\mathrm{q}}$, respectively, on the graph axes) for their native distributions against $\sigma_{\dot{V}}$ expressed as a function of $\dot{V} / \dot{Q}\left(\sigma_{\dot{V}} \mid \dot{V} / \dot{Q}\right)$, left and $\sigma_{\dot{Q}}$ expressed as a function of $\dot{V} / \dot{Q}\left(\sigma_{\dot{Q}} \mid \dot{V} / \dot{Q}\right)$, right (note: $\dot{V} / \dot{Q}$ is shown as v/q on the graph axes). 


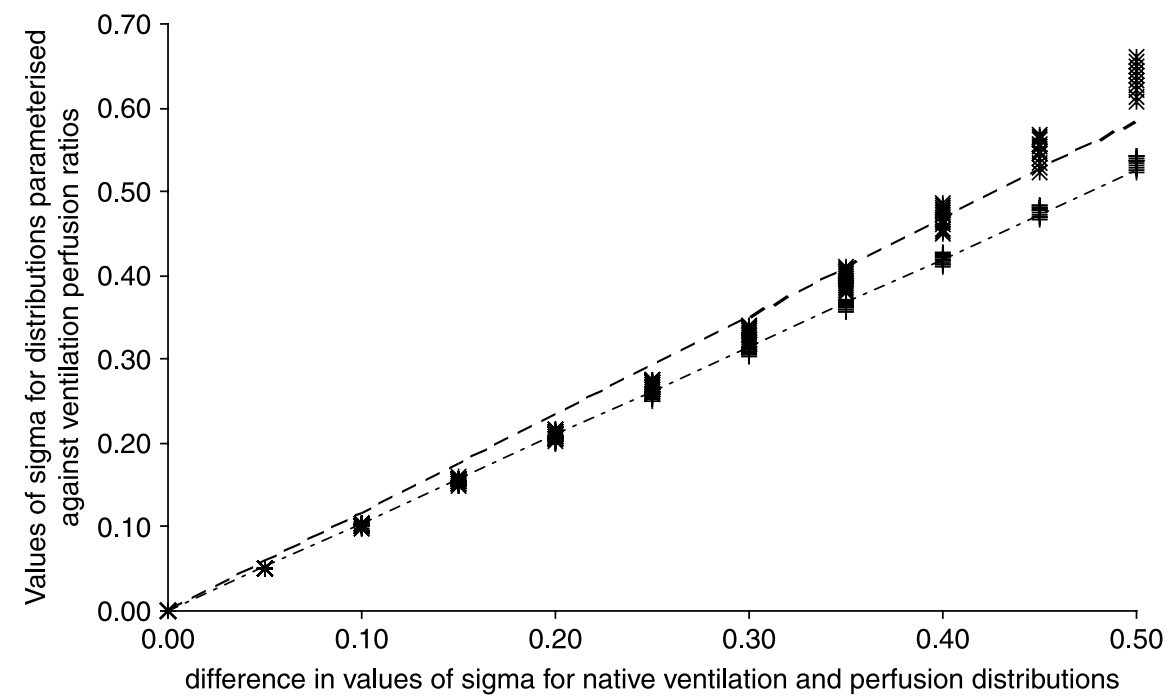

Figure 2. The relationship between the modulus of the difference in value of sigma for the native distributions of ventilation and perfusion $(|\sigma \dot{V}-\sigma \dot{Q}|)$ and the value of sigma obtained for ventilation as a function of $\dot{V} / \dot{Q}\left(\sigma_{\dot{V}} \mid \dot{V} / \dot{Q}\right)$ shown $(\times,--)$ and perfusion as a function of $\dot{V} / \dot{Q}$ $\left(\sigma_{\dot{Q}} \mid \dot{V} / \dot{Q}\right)$, shown $(+,-\cdot \cdot)$ for $\dot{V}_{T} / \dot{Q}_{T}=1$. In each case the line shown is the line of best fit.

value of $\sigma$ to the values of $\sigma_{\dot{V}} \mid \dot{V} / \dot{Q}$ and $\sigma_{\dot{Q}} \mid \dot{V} / \dot{Q}$, values of $\sigma_{\dot{V}} \mid \dot{V} / \dot{Q}$ and $\sigma_{\dot{Q}} \mid \dot{V} / \dot{Q}$ were calculated for $\left|\sigma_{\dot{V}}-\sigma_{\dot{Q}}\right|=0.35$ as the mean value of $\sigma$ was varied between 0.2 and 1.1 in steps of 0.1. The results, plotted in Figure 4, showed that the values of $\sigma_{\dot{V}} \mid \dot{V} / \dot{Q}$ and $\sigma_{\dot{Q}} \mid \dot{V} / \dot{Q}$ were largely independent of the mean value of $\sigma$. Thus, a mean value of 0.4 was selected so that $\left|\sigma_{\dot{V}}-\sigma_{\dot{Q}}\right|$ was evenly distributed about the mean value. This give values for $\sigma_{\dot{V}}$ and $\sigma_{\dot{Q}}$ of 0.23 and 0.58 , respectively. These are the dispersion parameters for the log normal distribution of ventilation and perfusion in a normal lung. Used with Equation (1), these will give the distribution of ventilation values at the end of the conducting airways and the distribution of perfusion values at the corresponding level within the pulmonary circulation.

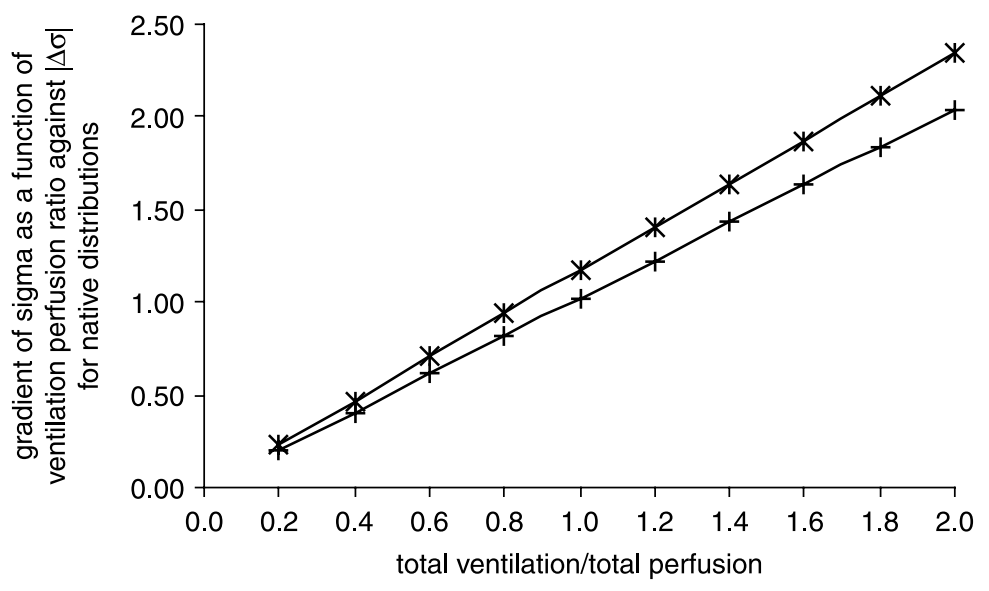

Figure 3. The gradient of the line of best fit for $\sigma_{\dot{V}} \mid \dot{V} / \dot{Q}$ against $\left|\sigma_{\dot{V}-} \sigma_{\dot{Q}}\right|=\Delta \sigma(\times)$ and for $\sigma_{\dot{Q}} \mid V / Q\left(\right.$ ) against $\left|\sigma_{\dot{V}-} \sigma_{\dot{Q}}\right|=\Delta \sigma(+)$ (see Figure 2) for values of $\dot{V}_{T} / \dot{Q}_{T}$ between 0.2 and 2 . 
Computational and Mathematical Methods in Medicine

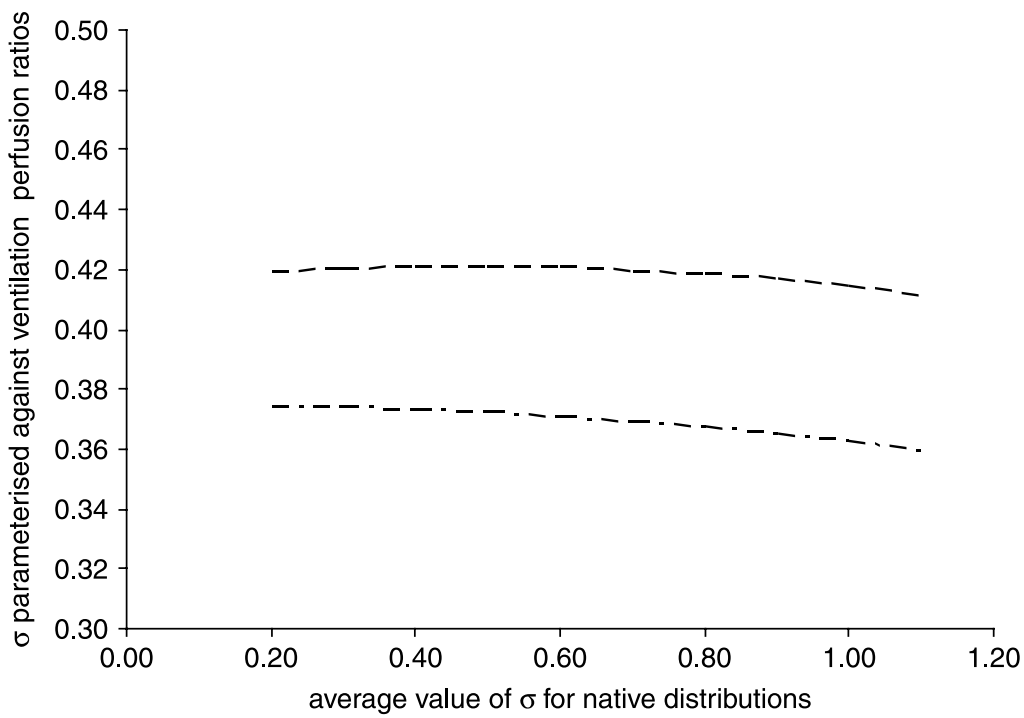

Figure 4. Plot showing the values of values of $\sigma_{\dot{V}} \mid \dot{V} / \dot{Q}\left(--^{--)}\right.$and $\sigma_{\dot{Q}} \mid \dot{V} / \dot{Q}\left(-\cdot{ }^{-}\right)$against average values of $\sigma_{\dot{V}}$ and $\sigma_{\dot{Q}}$ for their native distributions with $\left|\sigma_{\dot{V}_{-}} \sigma_{\dot{Q}}\right|=0.35$.

Having obtained values for $\sigma_{\dot{V}}$ and $\sigma_{\dot{Q}}$, it is now possible to generate two sets of numbers $\dot{V}_{i}$ and $\dot{Q}_{i}(i=1 \ldots N)$ for the $N$ compartments of the lung model from a log normal distribution with the parameter sets $\left(\mu_{\dot{V}}, \sigma_{\dot{V}}\right)$ and $\left(\mu_{\dot{Q}}, \sigma_{\dot{Q}}\right)$, respectively. A set of $\dot{V}_{i} / \dot{Q}_{i}$ ratios can then be obtained from the individual values using:

$$
\frac{\dot{V}}{\dot{Q}}=\frac{\dot{V}_{i}}{\dot{Q}_{i}}
$$

for $i=1, \ldots, N$. A $\chi^{2}$ analysis of the resultant histogram of these $\dot{V} / \dot{Q}$ ratios for $N=2000$, shows that this in turn is $\log$ normally distributed both when $\dot{V}_{i}$ and $\dot{Q}_{i}$ are correlated $(p=0.96)$ and when they are uncorrelated $(p=0.99)$.

\subsection{Structural models}

Whilst there are good theoretical reasons for the use of a log normal distribution there is a need to establish whether that distribution is appropriate to a model of the physical structure of the lung suitable for the investigation of acute lung disease. For the purposes of this study, the lung has been modelled as a dichotomously branching tree such that the flow at the top of the tree is normalized to 1 . This parent tube bifurcates into two daughter tubes and conservation of mass governs that if a fraction $\alpha$ flows down one daughter tube, a fraction $\beta=1-\alpha$ has to flow down the other. Each daughter tube now bifurcates with a fraction $\alpha$ of the flow in the parent tube flowing down one of the new daughter tubes and a fraction $\beta$ of the flow in the parent tube flowing down the other. An asymmetric flow distribution where $\alpha \neq \beta$ is a more realistic model of the branching structure in the human airways [10] than the symmetric model proposed by Weibel [25] that has often been used in flow analysis in the past. A whole tree with the same flow asymmetry at each generation is shown in Figure 5, together with the corresponding flow rates in each generation. 


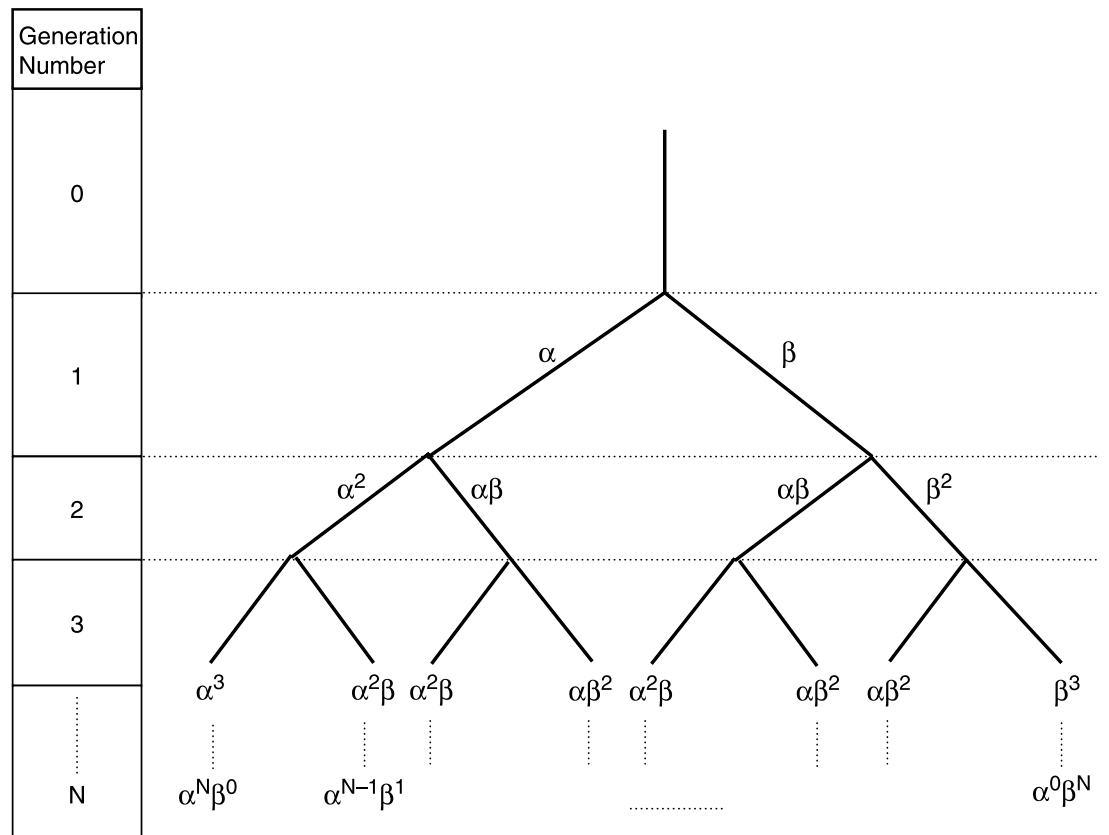

Figure 5. A bifurcating tree model of the human lung with unequal flow down the left $(\alpha)$ and right $(\beta)$ branches, respectively.

Normalizing the flow at the top of the branching tree structure to 1 eliminates the effects of the resistance of the airways, which will cause a change in the numerical values of the flows. The flow rates in the $2^{N}$ branches at the $N$ th generation of the tree are quantized to $N+1$ discrete values $\left(\dot{V}_{n k}\right)$ given by:

$$
\dot{V}_{n k}=\alpha^{n} \beta^{k}
$$

where $n=0, \ldots, N$ and $k=N-n$, and the number of occurrences of each discrete flow rate within a generation is given by:

$$
N_{\dot{V}_{n k}}=\frac{N !}{(N-k) ! k !} .
$$

The total flow at that $N$ th generation $\dot{V}_{N}$ is given by the product of Equations (7) and (8) summed over all unique flow values:

$$
\dot{V}_{N}=\sum_{n=0}^{N} N_{\dot{V}_{n k}} \dot{V}_{n k}=1 .
$$

The parameters $\alpha$ and $\beta$ parameterize the asymmetry in the flow down the airways. As predicted by Equations (7) and (8), the distribution of normalized flows in the terminal branches contain only a few possible values (Figure 6(a)). This quantization of values is unlikely to occur in the real lung. However, if instead of the same flow asymmetry at each bifurcation a slightly different, and randomly varying, asymmetry is used such that the 

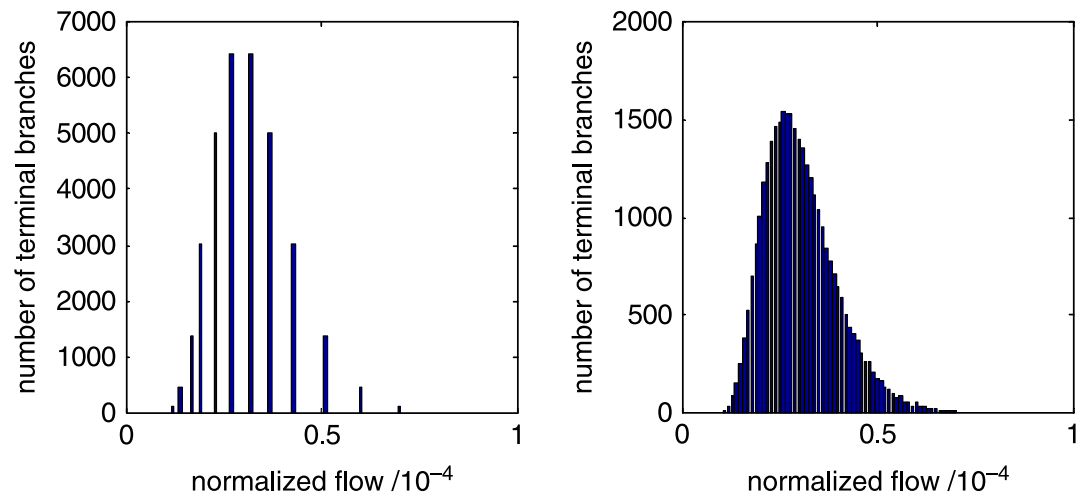

Figure 6. (a) Shows the distribution of terminal flows from a 15 level bifurcating tree with $\alpha=0.535$. (b) Shows the terminals flows for the same tree but where the flow at the nth branch is given by: $\alpha_{N}=0.51+0.05 \chi$, where $\chi$ is a random value from a uniform distribution on the interval $(0.0,1.0)$.

fraction of the flow going down one branch at the $n$th generation is given by:

$$
\alpha_{n}=\alpha \pm \eta \chi
$$

where $\alpha$ is the average flow asymmetry for the tree, $\chi$ is a random variable from a uniform distribution on the interval $(-0.5,+0.5)$ and $\eta$ is a constant with a value much smaller than $\alpha$. Using this, a larger number of terminal flows is created together with a more realistic model of the airways as shown in Figure 6(b). The parameter $\eta$ controls the amplitude of the random values that remove the quantization from the terminal flows and produces a smooth probability distribution. The magnitude of $\eta$ does not affect the overall shape of the distribution, but merely the number of values which define it. Therefore, the analysis is insensitive to its value provided it is much less than the flow asymmetry parameter, $\alpha$ which does control the shape of the distribution. Simulations showed that a value of $\eta$ of 0.025 gave smooth distributions for values of $\alpha$ between 0.51 and 0.6, and there was little improvement for values of $\eta$ greater than this.

The median and variance for the distribution of flow rates of the type shown in Figure 6(b) can be used to calculate the parameters $\mu$ and $\sigma$ for an equivalent log normal distribution from Equations (3) and (4). Applying these to the flow rates shown in Figure 6(b) and comparing this distribution with the equivalent log normal distribution using a $\chi^{2}$ analysis showed that the flow rates at the terminal branches of the bifurcating tree model are $\log$ normally distributed $(p=0.84)$.

Rather than use Equation (10) to produce a smooth distribution, it is also possible to transform the discrete distribution into a continuous one resulting in a distribution that is only dependant on $\alpha$ and $\beta$. The probability of a particular discrete flow rate $p(x)$, where $x=\alpha^{n} \beta^{k}$ occurring at the $N$ th level of the bifurcating tree model can be determined from the number of occurrences of each discrete value given by Equation (7) and the total number of branches which is $2^{N}$ :

$$
p(x)=\left(\frac{N !}{(N-k) ! k !} \frac{1}{2^{N}}\right) .
$$


If instead of integer values for $n$ and $k$, we assume that they are real values on the interval $(0, N)$ then given that $n !=\Gamma(n+1)$, where:

$$
\Gamma(y)=\int_{0}^{\infty} t^{y-1} \mathrm{e}^{-t} \mathrm{~d} t
$$

the probability density function corresponding to the new continuous (rather than discrete) random variable $x$ can be written as:

$$
\phi(x)=\frac{\Gamma(N+1)}{\Gamma(N-k+1) \Gamma(k+1)} \frac{1}{2^{N}} .
$$

Figure 7 shows a plot of $\phi(x)$ against $x$ for values of $\alpha$ between 0.52 and 0.60 . From the median and variance of the distribution for $\alpha=0.56$, the parameters $\mu$ and $\sigma$ for an equivalent log normal distribution were determined using Equations (3) and (4). This log normal distribution was then compared against the one obtained from the above equation using a $\chi^{2}$ analysis, which showed that the distribution of flow rates from the continuous transformation of the bifurcating tree are log normally distributed $(p=0.99)$. Therefore, flows in the terminal branches in the bifurcating tree model have a log normal distribution which is the distribution used to create the statistical model (Section 2.1). Figure 7 suggests that the modal value of the distribution decreases with increasing asymmetry, whilst the spread of values increases. A graph of $\sigma$ for the log normal distribution against the asymmetry parameter $\alpha$ shows that these are linearly related (Figure 8). The analysis of the log normal distributions for the compartmental models (Sections 1 and 2.1) yielded values of $\sigma_{\dot{V}}$ and $\sigma_{\dot{Q}}$ of 0.23 and 0.58 , respectively, for a normal adult lung. Using the data in Figure 8, the values for $\alpha$, the asymmetry parameter from the bifurcating tree model,

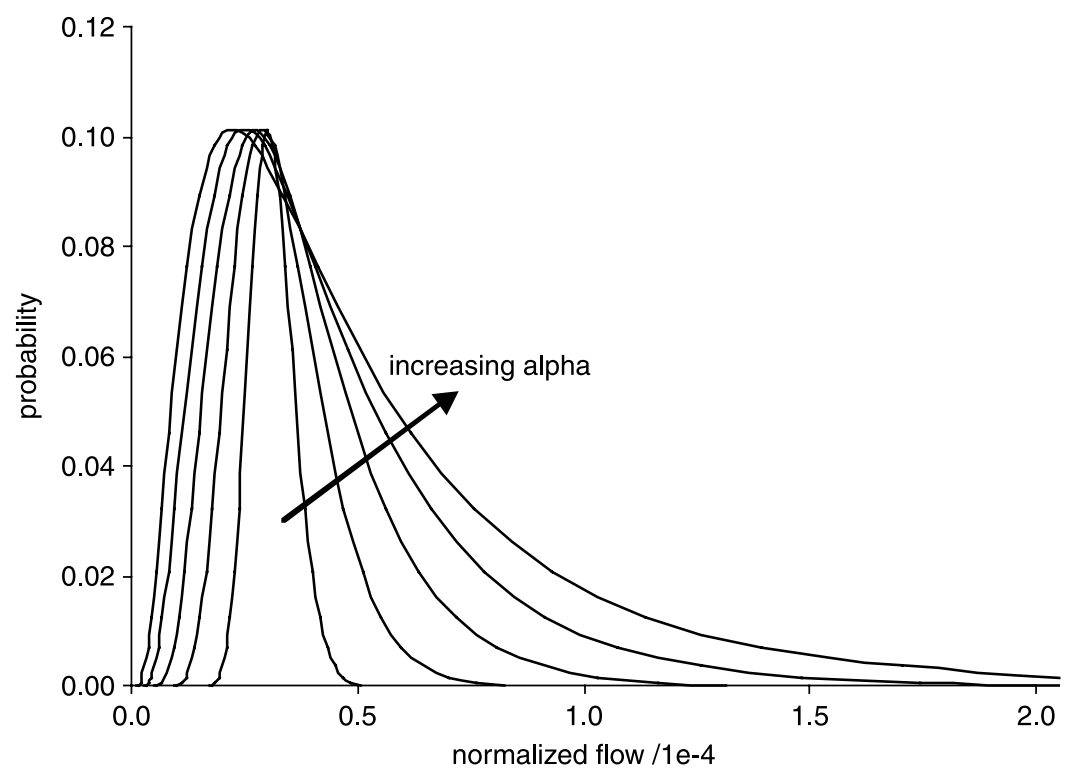

Figure 7. A series of distributions generated using a gamma function as the asymmetry between the left and right branches (gamma function parameter $\alpha$ ) is varied between 0.52 and 0.6. 


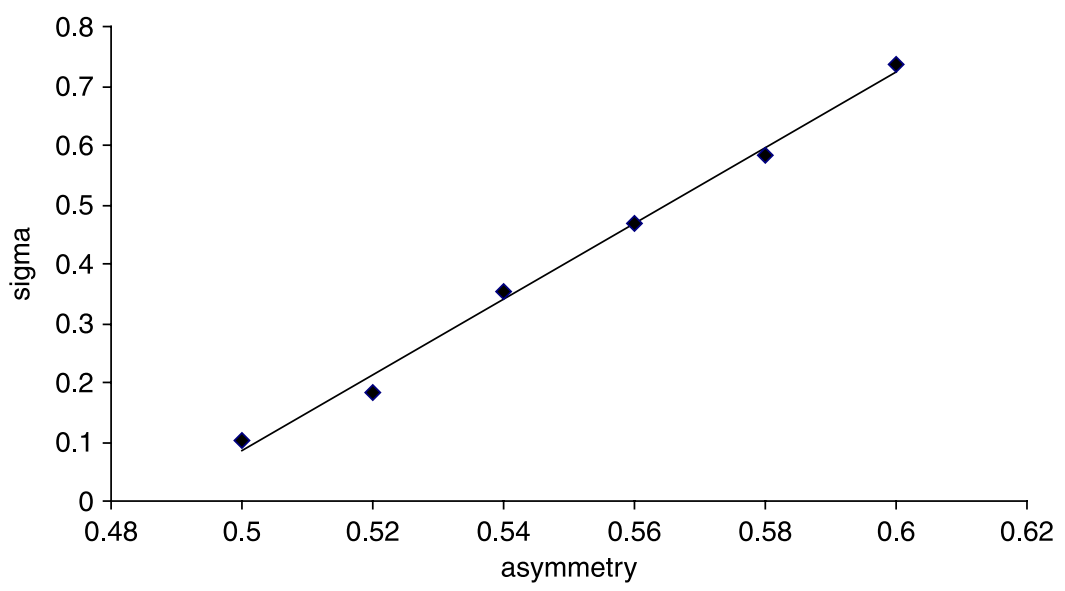

Figure 8. Plot showing the relationship between the asymmetry in the bifurcating tree (gamma function parameter $\alpha$ ) and the parameter $\sigma$ from the equivalent $\log$ normal distribution.

which will give a distribution of flows in the terminal branches with the same values of $\sigma_{\dot{V}}$ and $\sigma_{\dot{Q}}$ are $\alpha=0.53$ and 0.58 , respectively.

\section{Theoretical model for damaged lungs}

Having postulated a mathematical model for a normal lung, the next question to be addressed is: how can lung damage be quantified using these postulated models? Throughout the following discussion, it is assumed that the lung disease only affects the ventilation side of the lung and not the pulmonary circulation. In physiological terms, such a change could result from mucous plugging of the small airways.

\subsection{Simulating lung disease in the log normal data series model}

Assume that there is $D \%$ damage to the lung. There are two simple ways in which this ventilation damage can be represented by the log normal data series model of the $\dot{V} / \dot{Q}$ distribution:

(i) a reduction in ventilation to each compartment by $D \%$; and

(ii) a very large reduction in ventilation to a selected $D \%$ of the compartments.

Dantzker [7] demonstrated that patients suffering from adult respiratory distress syndrome (ARDS) tend, in general, to have bimodal distributions. Case (i) would certainly not have the effect of increasing the number of modes in the distribution of a normal lung. In addition, it would be inconsistent with the finding from patient studies that lung disease is spatially regional $[14,23]$. In order to analyse case (ii), we need to consider which $D \%$ of the compartments would become damaged and what the reduction in ventilation should be to each one of those compartments. The ventilation for all compartments form a population of sample size $N_{s}$ that are $\log$ normally distributed with the parameters $\mu_{\dot{V}}$ and $\sigma_{\dot{V}}$. Now, if a small fraction $d=D / 100$ of the compartments were randomly selected from the whole population, then that sample will also be log normally distributed with parameters $\mu_{\dot{V}}$ and $\sigma_{\dot{V}}$ provided $N_{s}$ is sufficiently large and that $N_{d}=d N_{S}$ is also a fairly large sample. If this sample of compartments were then damaged, so that the ventilation to 
them is reduced to a very small percentage of the original, then effectively the resultant series will contain two sub-series: one that represents the undamaged proportion of the lung and one that represents the damaged portion of the lung. In order to determine what percentage compartmental damage should be applied, it is necessary to determine how the appearance of a $\log$ normal distribution is affected by the percentage of compartments damaged and by values of $\sigma$. Using six $\log$ normally distributed data series from a random number generator all of which had a value of $\mu$ of 1 and with equi-spaced values of $\sigma$ between 0.15 and 0.65 , the percentage of compartments damaged was changed from 5 to $95 \%$ in $2.5 \%$ increments. For each value of the percentage of compartments damaged, the minimal compartmental damage was recorded when two peaks were just visible in the resultant distribution. An example of this process is shown in Figure 9, where the percentage of compartments damaged was set to $10 \%$ and $\sigma$ to 0.25 . In this case, bimodality was assessed to occur when the compartmental damaged was $\geq 52.5 \%$. The results of the analysis (Figure 10) show that the minimum compartmental damage necessary to create a bimodal distribution rises linearly with $\sigma$, that it is less than 0.85 for all values of $\sigma$ less than 0.60 and that it is largely independent of the percentage of compartments damaged.

\subsection{Simulating lung disease in the bifurcating tree model}

In the bifurcating tree model, damage to an area of the lung can be simulated by changing the value of $\alpha$ to $\alpha^{\prime}$ (the branch damage) at one particular branch such that the flow in that
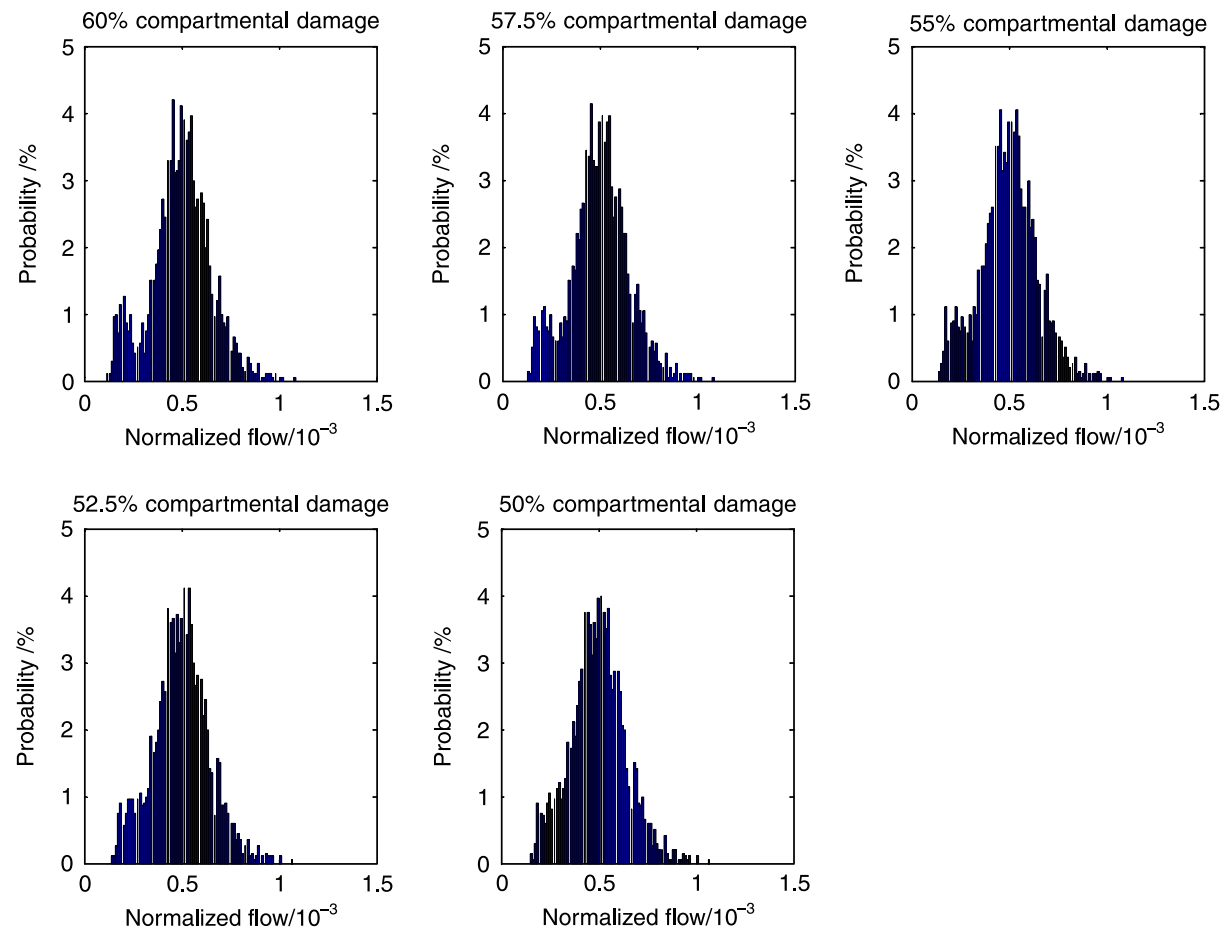

Figure 9. The probability distribution for flows in the log normal distribution model $(\sigma=0.25)$, where $10 \%$ of the compartments have been damaged and as the compartmental damage is reduced from 60 to $50 \%$. 


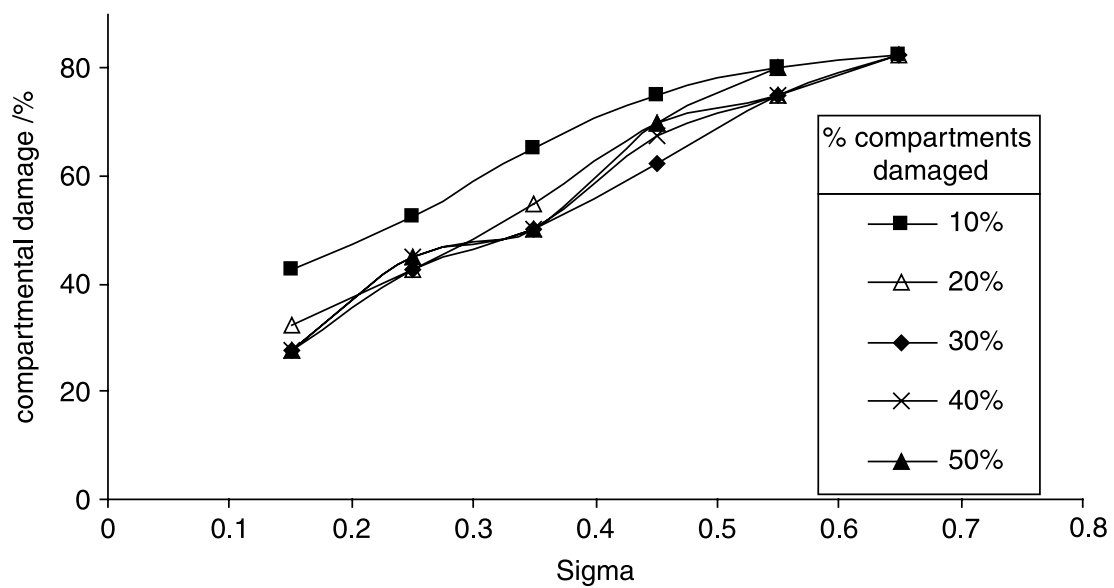

Figure 10. The minimal compartmental damage necessary to form a bimodal distribution as a function of the log normal parameter $\sigma$ and the percentage of compartments damaged.

daughter branch is reduced whilst the flow down the other daughter branch $\left(\beta^{\prime}=1-\alpha^{\prime}\right)$ will increase (Figure 11). This is analogous to the compartmental damage applied in the $\log$ normal data series experiment. By damaging multiple branches it is possible to simulate different degrees of lung damage which is analogous to the number of compartments damaged in the log normal data series model. In addition to modifying the flow within the tree and applying those modifications to multiple branches, the modifications can also be applied to different levels of the tree. To investigate the

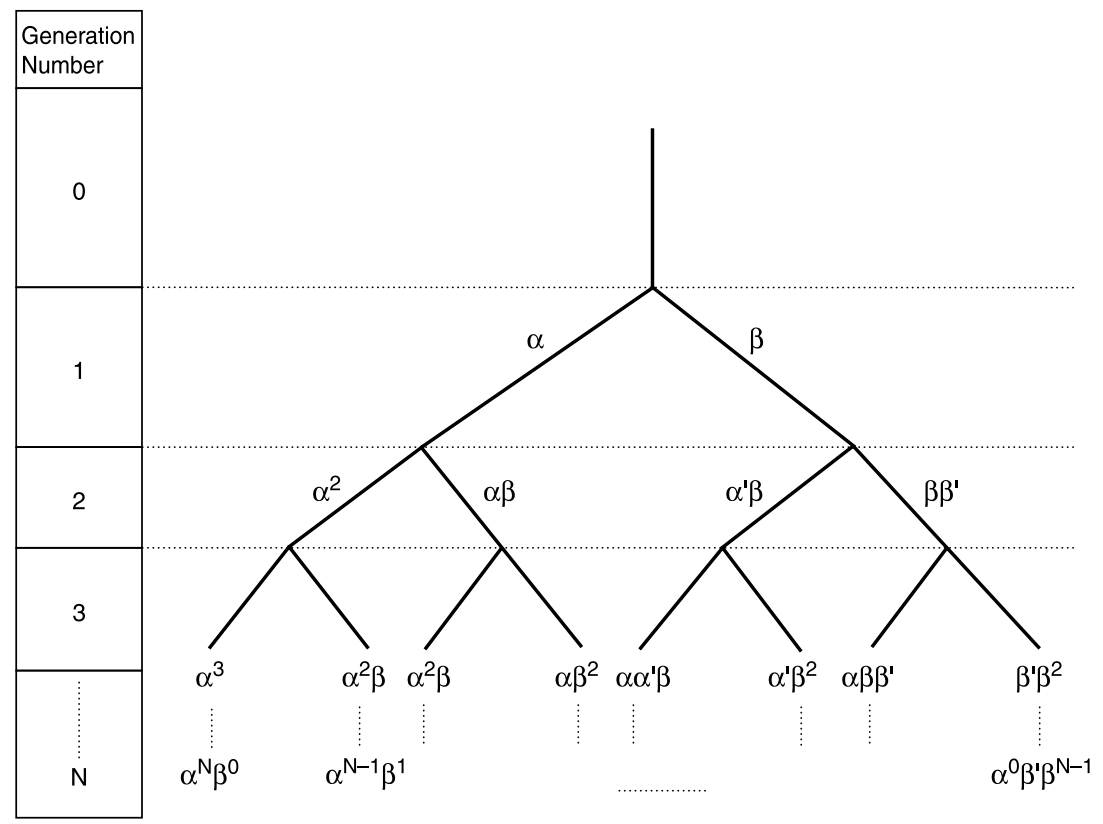

Figure 11. A bifurcating tree model of the human lung with damage at level 2. The flow in the left hand branch at the damage level is $\alpha^{\prime}$, whilst the flow in the right hand branch is $\beta^{\prime}=\left(1-\alpha^{\prime}\right)$. 


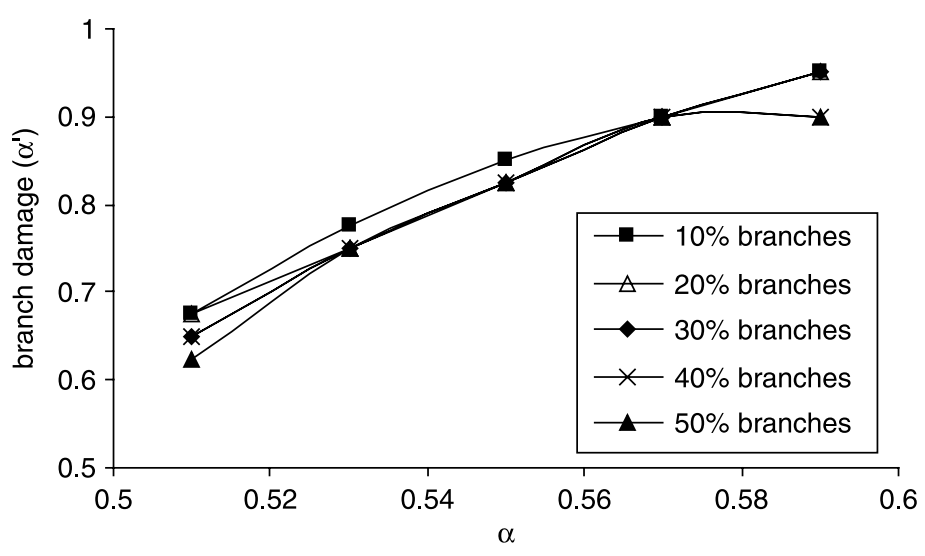

Figure 12. Plot of branch damage, $\alpha^{\prime}$, at tree level 14 required to produce a bimodal distribution in the terminal flows as a function of the average value of $\alpha$ and where the number of branches damaged varies between 10 and $50 \%$.

appearance of bimodality in the distribution of terminal flows from the tree, the branch damage at which bimodality occurred was found when this was applied to between 10 and $50 \%$ (in steps of 10\%) of the branches at a particular level, and when the average values of $\alpha$ was varied between 0.51 and 0.59 in steps of 0.2 . This analysis was repeated for damage to the tree at all levels between 10 and 15 . The bimodality of the resultant frequency distributions was identified visually from the plots of these. The results obtained showed that the branch damage $\left(\alpha^{\prime}\right)$ required to produce a bimodal distribution rose approximately linearly with the average value of $\alpha$ for the tree and was largely independent of the number of branches damaged (Figure 12). In addition, applying the patterns of flow modification at different level of the tree showed that these findings were independent of the level in the tree at which the modifications were applied. Analysis of the results showed that a value of branch damage greater than 0.95 will always produce a bimodal distribution independent of the value of $\alpha$, the number of branches where flow modification is applied and the level in the tree where that flow modification is applied. If it is assumed that the average value of $\alpha$ is 0.55 , then a branch damage of 0.95 corresponds to a flow modification of $90 \%$.

\section{Discussion}

In this paper, we have examined two theoretical models of the conducting airways of the lung: a log normal data series model and a bifurcating tree model. Using the log normal data series model we have shown that if the distributions of $\dot{V}$ and $\dot{Q}$ are $\log$ normally distributed, then the $\dot{V} / \dot{Q}$ ratio is also log normally distributed. In doing this, we have established a method for determining values of $\sigma_{\dot{V}}$ and $\sigma_{\dot{Q}}$ based on distribution values that can be obtained from MIGET experiments. In addition, we have shown that $\left|\sigma_{\dot{V}}-\sigma_{\dot{Q}}\right|$ is linearly related to $\sigma_{\dot{V}} \mid \dot{V} / \dot{Q}$ and $\sigma_{\dot{Q}} \mid \dot{V} / \dot{Q}$ for values of $\sigma_{\dot{V}}<0.5$. Colburn et al. [6] showed theoretically that that this relationship was a function of $\left|\sigma_{\dot{V}}-\sigma_{\dot{Q}}\right|$ for the case where the mean value of ventilation and perfusion were the same. In this paper, we have also shown that the value of $\left|\sigma_{\dot{V}}-\sigma_{\dot{Q}}\right|$ increases linearly with the ratio of total ventilation to total perfusion. Wilson and Beck [27] approached the problem of determining values for $\sigma_{\dot{V}}$ and $\sigma_{\dot{Q}}$ in supine dogs using a hyperplane technique applied to the results from MIGET experiments. Using the results obtained in this paper, together with the published values 
from studies on normal subjects using the MIGET technique, possible values of $\sigma_{\dot{V}}$ and $\sigma_{\dot{Q}}$ of 0.23 and 0.58 , respectively, were obtained. Caution must be exercised in interpreting and using these values, since they were obtained using the results from MIGET experiments for a small number of subjects using early revisions of the parameter recovery algorithms. There is little information on how these distributions may vary with age. Wagner et al. [24] suggested that there was a consistent broadening of the $\dot{V} \mid \dot{V} / \dot{Q}$ and $\dot{Q} \mid \dot{V} / \dot{Q}$ distributions with increasing age for normal subjects, but they made measurements on only 12 normal subjects whose ages ranged from 21 to 60 . This is not a sufficiently large enough sample to establish an exact relationship.

Using data series of random numbers with log normal distributions to represent values of $\dot{V}$ and $\dot{Q}$ in the compartments of a multi-compartment lung model, it was shown that the resultant $\dot{V} / \dot{Q}$ distributions are $\log$ normally distributed both when $\dot{V}$ and $\dot{Q}$ are correlated and when they are uncorrelated. It is generally assumed that ventilation and perfusion are correlated and that the matching between ventilation and perfusion is actively maintained $[3,21]$. However, when lung damage was induced in an animal model, plots of $\dot{V}$ against $\dot{Q}$ showed an increased spread around the straight line $\dot{V} / \dot{Q}=2$ when compared with animals without lung damage [1]. In the model of lung disease proposed in this paper, lung damage is regional and therefore, there will be areas of the lung where ventilation and perfusion are either no longer correlated or where the correlation coefficient is very different from other parts of the lung. This is consistent with the finding in the animal model [1] of an increased spread of points on plots of $\dot{V}$ against $\dot{Q}$ when lung disease is present.

In terms of the bifurcating tree model of the conducting airways of the lung, the results show that the distribution of the terminal branch flow rates have log normal distributions when the branching is asymmetric. It should be noted that it is the asymmetric nature of the tree that yields these distributions since symmetric branching would result in equal flow rates at each terminal branch. Furthermore, we have shown that there is a linear relationship between the asymmetry factor $\alpha$ and the parameter $\sigma$ for the $\log$ normal distribution of terminal flows. A theoretical analysis of a bifurcating tree model of the pulmonary blood flow with fractal dispersion showed that the distribution of flows from such models were inherently log normally distributed [19]. Simulations have also been reported for electrical analogues of bifurcating networks with fractal characteristics [8], where the networks were composed of pathways having random Gaussian resistances. Constant potential sources applied to these networks, yielded log normal distributions of potentials at the terminal nodes. Thus, basing a model for ventilation and perfusion distributions in a normal lung on branching tree structures such as those described in this paper seems a reasonable basis for modelling lung disease. In the proposed model, the division of flow at each bifurcation is characterized by an asymmetry factor $\alpha$. In reality, the proportion of flow in the parent tube that is directed into each of the daughter tubes will depend on the length, diameters and branching angles of the daughter tubes, which determine the resistance to flow in each branch. Within this study, the distribution of flows in the terminal branches of the bifurcating tree model were compared with the equivalent $\log$ normal distribution using values of $\sigma_{\dot{V}}=0.23$ and $\sigma_{\dot{Q}}=0.58$ obtained from the distribution analysis. This yielded values for $\alpha$ of 0.53 and 0.58 for the ventilatory and pulmonary trees, respectively. In the case of the airways, these values of $\alpha$ are compatible with those obtained via flow simulations in geometries reconstructed from 3D medical images [12] and from cast reconstructions [18]. Whilst the values obtained for $\alpha$ are compatible with those from other studies, the bifurcating tree is only an approximation for the structure of the lung and a complete picture of the flow distribution for an individual 
subject can only be obtained using 3D CFD models with the geometry determined from anatomically accurate medical images [12].

A consistent finding in the literature is that $\dot{V} / \dot{Q}$ distributions become bimodal in the presence of lung disease. There is no standard definition of bimodality in a statistical distribution nor a standard test for its presence. For the purpose of the analysis presented in this paper bimodality was defined as the existence of two distinct peaks in the distribution identified by visual inspection.

The results from modelling lung damage by reducing the values of a randomly selected subset of values in a log normal data series representing the ventilation in the terminal airways of a multi-compartment lung showed that a bimodal distribution always resulted when the compartmental damage level was greater than $85 \%$. For a given percentage of compartments damaged, the fractional reduction in alveolar ventilation in the damaged compartments rises linearly with fractional reduction in the ventilation. Therefore, a high value of the fractional reduction in the compartmental ventilation for the damaged compartments results in the fraction of damaged cells approaching the fractional reduction in alveolar ventilation. There is no inherent way of allocating specific values of ventilation and perfusion modelled using a log normal random number series to a particular region of the lung. Therefore, if this approach to modelling lung disease is to be used a separate algorithm will be required to assign the compartmental values to mimic the spatial distribution of the disease within the lung.

In the bifurcating tree model of the conducting airways, lung damage was simulated by altering the flow in one or more branches of the tree. It should be noted that since the total flow is normalized to 1 , this process only produces a re-distribution of flow in the tree and not a change in the total flow through the airways. The finding that the level of flow alteration at which the distribution of terminal flows became bimodal was independent of the number of branches and the level in the tree where flow alteration applied was unexpected. This finding offers the potential of modelling a number of different regions of disease within the lung of different sizes, whilst producing distributions of the terminal flows that are consistent with those obtained from MIGET and other experimental techniques.

It is interesting to note that the level of damage required to always produce a bimodal distribution from the log normal data series model is $85 \%$ whilst the flow re-distribution required to always produce a bimodal distribution in the bifurcating tree model was $90 \%$. Given the lack of a formal test for bimodality in a distribution these values can be considered very similar and emphasize the similarity of the two approaches.

The models proposed in this paper offer the opportunity of developing realistic models of the effects lung disease has on the conducting airways and the pulmonary circulation and the potential for incorporating these into larger scale models of the respiratory and cardiovascular systems.

\section{Acknowledgements}

This research was funded by EPSRC under grants GR/L74323 and GR/L74835.

\section{References}

[1] W.A. Altemeier, H.T. Robertson, and R.W. Glenny, Pulmonary gas-exchange analysis by using simultaneous deposition of aerosolized and injected microspheres, J. Appl. Physiol. 85 (1998), pp. 2344-2351. 
[2] W.A. Altemeier, H.T. Robertson, S. McKinney, and R.W. Glenny, Pulmonary embolization causes hypoxemia by redistributing regional blood flow without changing ventilation, J. Appl. Physiol. 85 (1998), pp. 2337-2343.

[3] W.A. Altemeier, S. McKinney, and R.W. Glenny, Fractal nature of regional ventilation distribution, J. Appl. Physiol. 88 (2000), pp. 1551-1557.

[4] T.C. Amis, A.B.H. Crawford, A. Davidson, and L.A. Engel, Distribution of inhaled ${ }^{99 m}$ technetium-labelled ultrafine carbon particle aerosol (Technegas) in human lungs, Eur. Respir. J. 3 (1990), pp. 679-685.

[5] J.B. Bassingthwaighte and R.P. Beyer, Fractal correlation in hetereogeneous systems, Physica D 53 (1991), pp. 71-84.

[6] W.E. Colburn, J.W. Evans, and J.B. West, Analysis of the effects of solubility of gas exchange in non-homogeneous lungs, J. Appl. Physiol. 37 (1974), pp. 547-551.

[7] D.R. Dantzker, C.J. Brook, P. Dehart, J.P. Lynch, and J.G. Weg, Ventilation-perfusion distributions in the adult respiratory distress syndrome, Am. Rev. Respir. Dis. 120 (1979), pp. 1039-1052.

[8] L. DeArcangelis, S. Redner, and A. Coniglio, Anomalous voltage distribution of random resistor networks and a new model for the backbone at the percolation threshold, Phys. Rev. B 31 (1985), pp. 4725-4727.

[9] R.W. Glenny, S.L. Bernard, and H.T. Robertson, Pulmonary blood flow remains fractal down to the level of gas exchange, J. Appl. Physiol. 89 (2000), pp. 742-748.

[10] K. Horsfield and G. Cumming, Angles of branching and diameters at branches in the human bronchial tree, Bull. Math. Biophys. 29 (1967), pp. 219-235.

[11] C. Lenfant and T. Okubo, Distribution function of pulmonary blood flow and ventilationperfusion ration in man, J. Appl. Physiol. 24 (1968), pp. 668-677.

[12] S. Ley, D. Mayer, B.S. Brook, E.J.R. van Beek, C.P. Heusell, D.R. Hose, D. Rinck, and U.H. Kauczor, Radiological imaging as the basis for a simulation software of ventilation in the tracheo-bronchial tree, Eur. Radiol. 12 (2002), pp. 2218-2228.

[13] Y. Liu, Y.M.C. So, and C.H. Zhang, Modeling the bifurcating flow in the human lung airway, J. Biomech. 35 (2002), pp. 465-473.

[14] J.M. Luce, Acute lung injury and the acute respiratory distress syndrome, Crit. Care Med. 2 (1998), pp. 369-376.

[15] B. Mauroy, M. Filoche, J.S. Andrade, and S. Sapoval, Interplay between geometry and flow in an airway tree, Phys. Rev. Lett. 90 (2003), p. 188101.

[16] S.M. Mijailovich, S. Treppo, and J.G. Venegas, Effects of lung motion and tracer kinetics corrections on PET imaging of pulmonary function, J. Appl. Physiol. 82 (1997), pp. $1154-1162$.

[17] T.R. Nelson and D.K. Manchester, Modeling lung morphologies using fractal geometries, IEEE Trans. Med. Imaging 7 (1988), pp. 321-327.

[18] C.G. Phillips, S.R. Kaye, and R.C. Schroter, A diameter-based reconstruction of the branching pattern of the human bronchial tree. Part I. Description and application, Respir. Physiol. 98 (1994), pp. 193-217.

[19] H. Qian and J.B. Bassingthwaighte, A class of flow bifurcation models with log normal distribution and fractal dispersion, J. Theor. Biol. 205 (2000), pp. 261-268.

[20] H.T. Robertson, R.W. Glenny, D. Stanford, L.M. McInnes, D.L. Lutchel, and D. Covert, Highresolution maps of regional ventilation utilizing inhaled fluorescent microspheres, J. Appl. Physiol. 82 (1997), pp. 943-952.

[21] B.A. Simon, K. Tsusaki, and J.G. Venegas, Changes in regional lung mechanics and ventilation distribution after unilateral pulmonary artery occlusion, J. Appl. Physiol. 82 (1997), pp. 882-891.

[22] S. Treppo, S.M. Mijailovich, and J.G. Venegas, Contributions of pulmonary perfusion and ventilation to heterogeneity in $\dot{\mathrm{V}}_{\mathrm{A}} / \dot{\mathrm{Q}}$ measured by PET, J. Appl. Physiol. 82 (1997), pp. $1163-1176$.

[23] J. Varon and O.C. Wenker, The acute respiratory distress syndrome: Myths and controversies, Internet J. Emerg. Intensive Care Med. (1998). Available online at: www.ispub.com/journals/ IJEICM/Vol1N1/ards.htm

[24] P.D. Wagner, R.B. Laravuso, R.R. Uhl, and J.B. West, Continuous distributions of ventilationperfusion ratios in normal subjects breathing air and 100\% $\mathrm{O}_{2}$, J. Clin. Invest. 54 (1974), pp. 54-68. 
[25] E.R. Weibel, Morphology of the Human Lung, Springer-Verlag, Berlin, 1963.

[26] J.B. West and P.D. Wagner, Pulmonary gas exchange, in Bioengineering Aspects of the Lung, J.B. West, ed., Marcel Decker Inc., New York, 1977.

[27] T.A. Wilson and K.C. Beck, Contribution of ventilation and perfusion inhomogeneities to the $\mathrm{V}_{\mathrm{A}} / \mathrm{Q}$ distribution, J. App. Physiol. 72 (1992), pp. 2298-2304.

[28] J.H.G.M. van Beek, S.A. Roger, and J.B. Bassingthwaighte, Regional myocardial flow heterogeneity explained with fractal networks, Am. J. Physiol. 257 (1989), pp. H1670-H1680. 


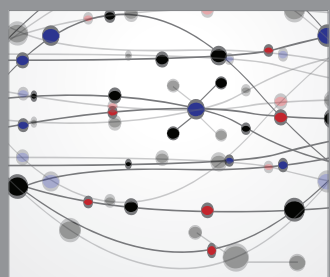

The Scientific World Journal
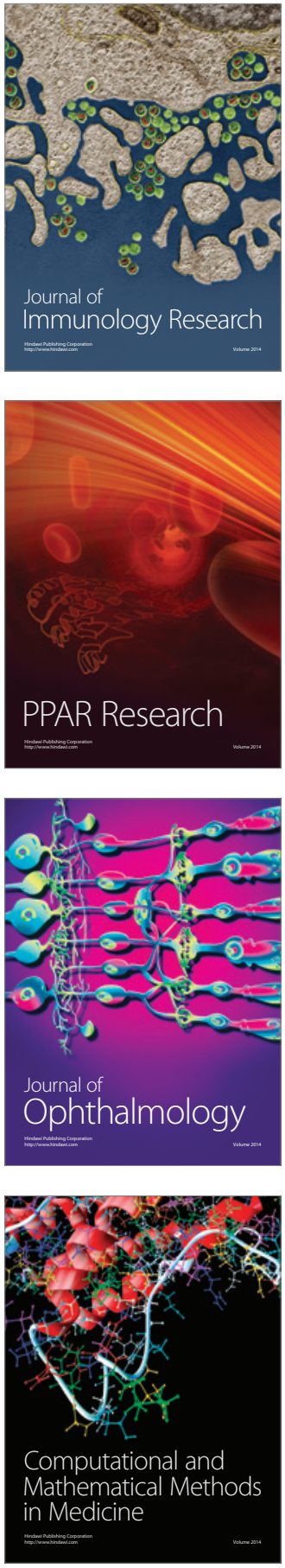

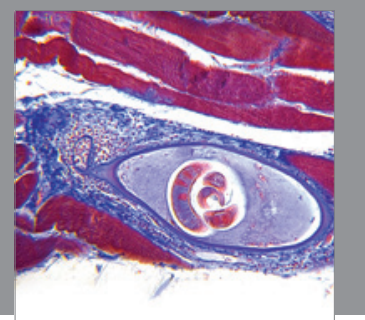

Gastroenterology

Research and Practice
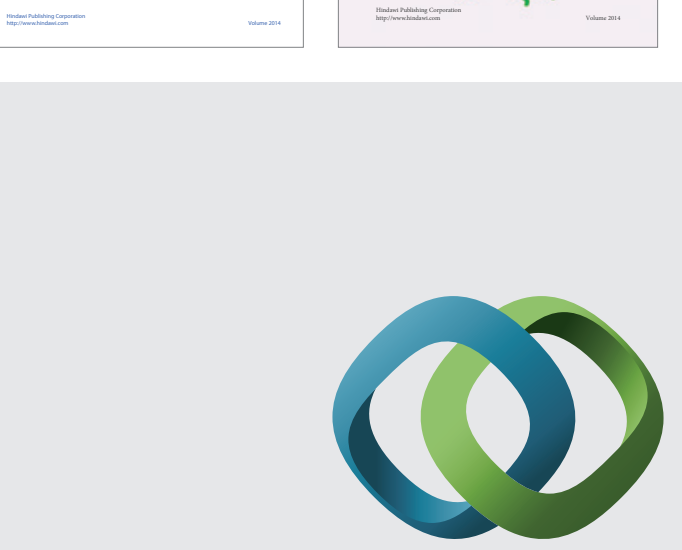

\section{Hindawi}

Submit your manuscripts at

http://www.hindawi.com
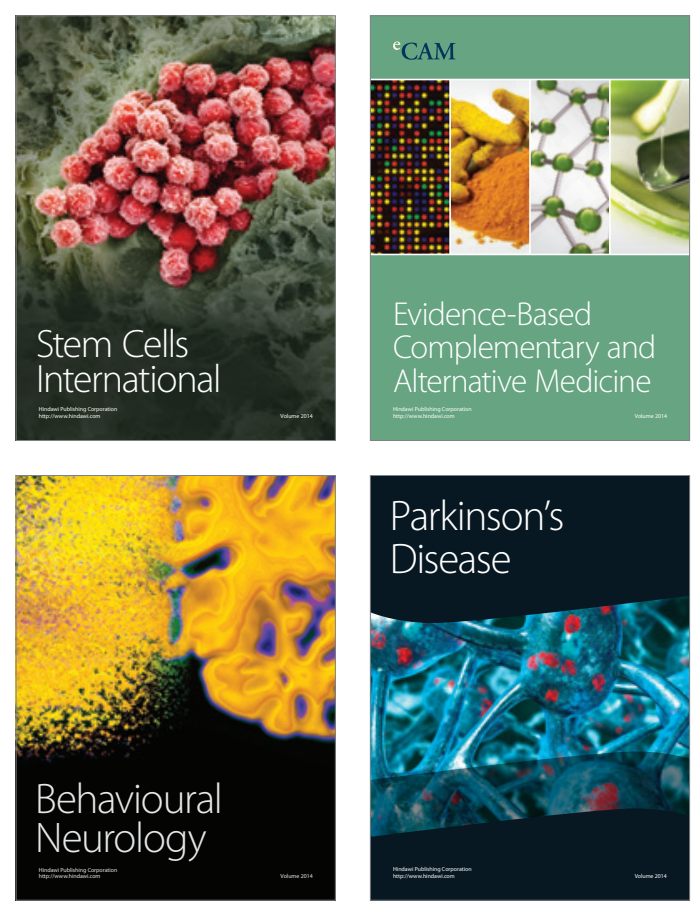

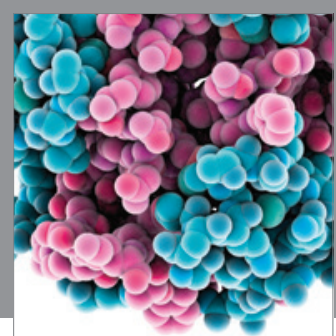

Journal of
Diabetes Research

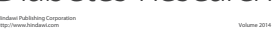

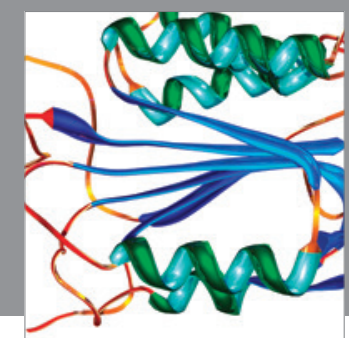

Disease Markers
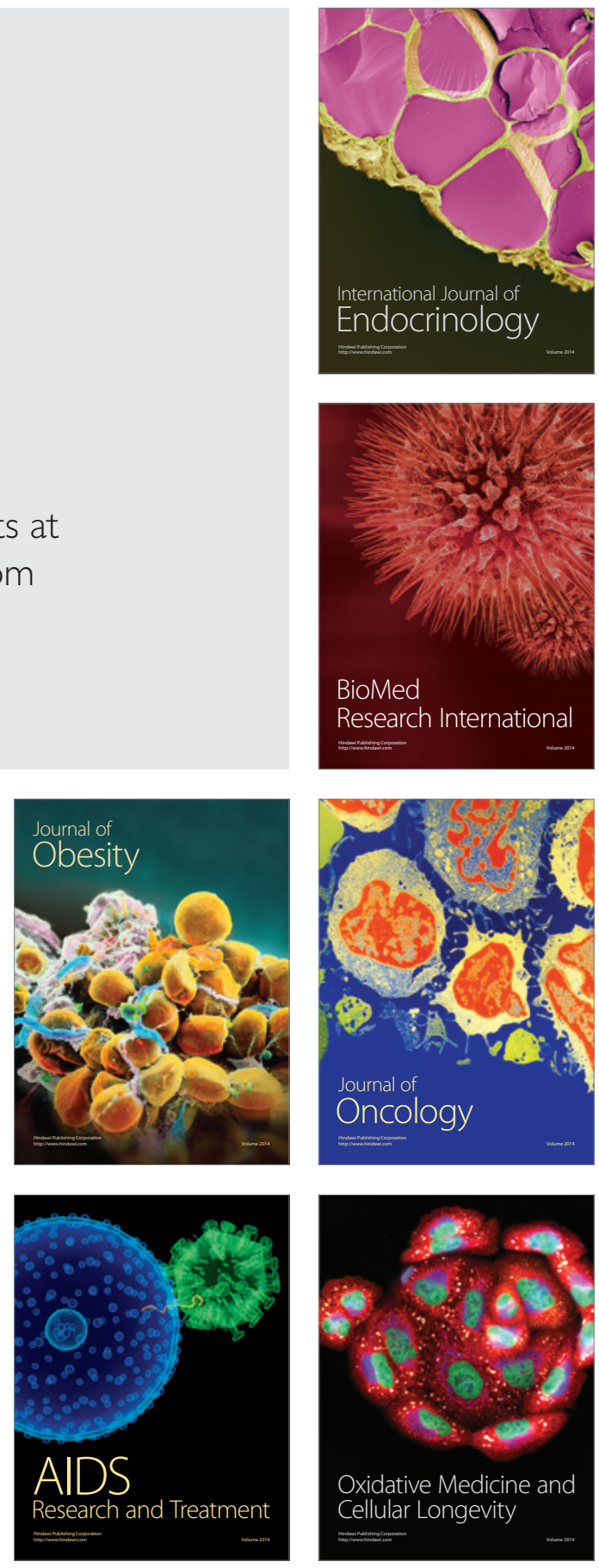